\title{
Correlation between Serum Fatty Acid Binding Protein 4 (FABP4) Levels and Cardiac Function in Patients with Thalassemia Major
}

\author{
Pandji I. Fianza $\mathbb{D}^{1,2,3}$ Anita Rahmawati $\mathbb{D}^{2},{ }^{2}$ Shofura Afifah, ${ }^{2}$ Suhendra Praptama, \\ Mohammad Ghozali $\left(\mathbb{1}^{2,4}\right.$ Teddy A. Sihite $\left(\mathbb{0},{ }^{5}\right.$ Djatnika Setiabudi $\oplus^{\circ},{ }^{6}$ \\ Mas R. A. A. Syamsunarno $\mathbb{D}^{2,4}$ Suthat Fucharoen $\mathbb{1}^{7},{ }^{7}$ and Ramdan Panigoro ${ }^{2,4}$ \\ ${ }^{1}$ Department of Internal Medicine, Division of Hematology and Medical Oncology, Faculty of Medicine, Universitas Padjadjaran/ \\ Hasan Sadikin General Hospital, Bandung, West Java, Indonesia \\ ${ }^{2}$ Research Center of Medical Genetics, Faculty of Medicine, Universitas Padjadjaran, Bandung, West Java, Indonesia \\ ${ }^{3}$ Doctoral Study Program, Faculty of Medicine, Universitas Padjadjaran, Bandung, West Java, Indonesia \\ ${ }^{4}$ Department of Biomedical Sciences, Faculty of Medicine, Universitas Padjadjaran, Bandung, West Java, Indonesia \\ ${ }^{5}$ Department of Cardiology and Vascular Medicine, Faculty of Medicine, Universitas Padjadjaran/Hasan Sadikin General Hospital, \\ Bandung, West Java, Indonesia \\ ${ }^{6}$ Department of Child Health, Faculty of Medicine, Universitas Padjadjaran/Hasan Sadikin General Hospital, Bandung, \\ West Java, Indonesia \\ ${ }^{7}$ Thalassemia Research Center, Institute of Molecular Biosciences, Mahidol University, Nakhonpathom, Thailand
}

Correspondence should be addressed to Mas R. A. A. Syamsunarno; rizky@unpad.ac.id

Received 24 April 2021; Revised 10 October 2021; Accepted 4 December 2021; Published 27 December 2021

Academic Editor: Heng Zhou

Copyright (c) 2021 Pandji I. Fianza et al. This is an open access article distributed under the Creative Commons Attribution License, which permits unrestricted use, distribution, and reproduction in any medium, provided the original work is properly cited.

\begin{abstract}
Background. Patients with thalassemia major may suffer from complications due to iron overload. It has been suggested that several adipokines may play a potential role in the development of complications in thalassemia. Fatty acid-binding protein 4 (FABP4) is one of the adipokines, bridging several aspects of metabolic and inflammatory pathways. Little is known about the relationship between this adipokine and cardiac and liver function, especially in patients with thalassemia major. Aims. This study is aimed at determining serum FABP4 levels in patients with thalassemia major and whether its concentration correlated with serum ferritin levels, as well as cardiac and liver function. Methods. Thalassemia major outpatients $(n=48)$ completed laboratory examination, echocardiography, and electrocardiography. Results. The mean age was $21.9 \pm 8.0$ years. A negative and weak correlation between serum ferritin and FABP4 was observed $(r=-0.291, p<0.05)$. In addition, there was moderate and positive correlation between left atrial volume index (LAVI) and FABP4 $(r=0.316, p<0.05)$. Conclusions. Serum FABP4 correlated with serum ferritin and cardiac function in patients with thalassemia major. FABP4 may be a potential clinical biomarker for cardiac dysfunction via metabolic and inflammatory pathways due to iron accumulation and toxicity in patients with thalassemia major.
\end{abstract}

\section{Introduction}

Thalassemia is one of the most common hereditary diseases that interfere with the synthesis of the normal globin chain, occurring in no less than 2.4 out of every 1000 live births, globally [1-3]. Ineffective erythropoiesis and hemolysis encompass the main reason of anemia in thalassemia [3, 4]. Severe anemia from early life is characteristic of thalassemia major thus requiring routine blood transfusion to survive [4]. However, a continuous load of iron due to repeating transfusion will result in iron overload because the body has no active elimination pathway for iron $[5,6]$. 
Therefore, to reduce iron accumulation and its related complications, iron chelation therapy is needed $[7,8]$.

The report suggests that in thalassemia, the liver is the primary target organ dysfunction, augmented by initiation of free radical reactions due to iron overload-induced oxidative stress [9, 10]. Delayed in diagnosis and treatment contributed to development of liver iron overload into cirrhosis [11]. On the other hand, among complications of iron overload, 63.6-71\% of morbidity and mortality were affected by cardiomyopathy $[12,13]$. Cardiac arrhythmias and congestive heart failure have been recognized as the most common cause of death in thalassemia $[8,12,14-16]$.

Iron is one of the essential minerals for cellular function; however, iron overload, as happened in thalassemia patients, may result in cell injury $[12,17]$. Iron could initiate free radical reaction and potentially impair cellular metabolism, including carbohydrates, proteins, lipids, and nucleic acids $[5,17,18]$. A chronic inflammatory condition existed in patients with thalassemia major [19-21]. Inflammatory state resulted from reactive oxygen species production, lipid peroxidation, and other metabolic changes due to iron toxicity are risk factors for cardiac dysfunctions, such as heart failure and left ventricular dysfunction [22].

Fatty acid-binding proteins (FABPs) are a group of proteins that adjust intracellular lipid transport [23]. FABPs have molecular weights of $14-15 \mathrm{kDa}$ and have high-affinity bonding with hydrophobic ligands, including unsaturated and saturated fatty acids [24]. Among FABPs, fatty acidbinding protein 4 (FABP4), known as adipocyte FABP, is one of the adipokines that act as a novel biomarker for the metabolic and inflammatory state [25], and it may be affected in patients with thalassemia major because of the iron excess. FABP4 is mostly expressed in adipocytes and macrophages and is associated with several aspects of the metabolic and inflammatory pathway, such as metabolic syndrome and cardiovascular disease [26]. Elevation of FABP4 levels may play a role in left ventricular dysfunction [22]. It is also associated with metabolic syndrome, type 2 diabetes, nonalcoholic steatohepatitis, atherogenic dyslipidemia, and heart failure [27].

It has been reported that several adipokines may have a potential role in the pathophysiological mechanism of complications in thalassemia [19-21, 28, 29]. However, the study about association of FABP4 and cardiac function in patients with thalassemia major is still limited. Therefore, the objectives of this study were to evaluate the serum FABP4 levels in patients with thalassemia major and whether its concentration was affected by iron excess assessed by serum ferritin levels. Given the increasing evidence of iron toxicity-induced inflammation-related metabolic and cardiovascular disturbances, the relationships between FABP4 and function of the target organ, including heart and liver were also investigated.

\section{Materials and Methods}

2.1. Subjects. The study subjects were the family members of the Thalassemia Parents Association in Bandung, West Java, Indonesia. Between June and October 2017, an explanation and invitation were sent to patients with thalassemia major who regularly visited the outpatient clinics at the Department of Internal Medicine, Division of Hematology and Medical Oncology, Hasan Sadikin General Hospital, Bandung. Consecutive agreements from 48 subjects were received. Patients, who were older than 14 years old and have received transfusion for at least 2 years, were included in the study. Patients on acute and/or severe infection were excluded by history taking and physical examination to prevent interference of the results of serum ferritin [30]. This research study was undertaken with the understanding and written informed consent of each patient and with the approval of the Ethics Committee of Faculty of Medicine, Universitas Padjadjaran, Bandung (approval number: 662/ UN6.C10/PN/2017). This study conformed to the principles outlined in the Declaration of Helsinki.

Diagnosis of thalassemia was based on the clinical history and laboratory confirmation. Patients' data were retrieved from medical records. Upon obtaining written informed consent, age and sex were recorded. Additionally, the detailed variables regarding age at the start of transfusion and the interval of blood transfusion were also obtained based on patients' information. All patients had received chelating drugs, including deferasirox $(20 \mathrm{mg} / \mathrm{kg})$, deferiprone $(75 \mathrm{mg} / \mathrm{kg})$, or deferoxamine $(30-50 \mathrm{mg} / \mathrm{kg})$ either as monotherapy or combination. All subjects underwent blood and cardiac examination, including electrocardiography (ECG) and echocardiography.

2.2. Measurement of Laboratory Parameters. Before transfusion, after overnight fasting, in the morning, blood was collected through venipuncture from each patient and centrifuged for 15 minutes at $3,000 \mathrm{rpm}$ to separate the serum. Blood parameters, including pretransfusion hemoglobin, were measured using an automated hematology analyzer (Sysmex XN- ${ }^{\circledR}$ Series ${ }^{\mathrm{TM}}$ Hematology Analyzers). Serum ferritin level was assessed by the electrochemiluminescence immunoassay (ECLIA) method using ADVIA Centaur XPT Immunoassay System Siemens Healthineers. Although the specificity of serum ferritin is sometimes problematic for the estimation of body iron [5], it may expect siderosis when it is $>2500 \mathrm{ng} / \mathrm{mL}[13,30]$. The concentration of liver enzymes, including aspartate transaminase (AST) and alanine transaminase (ALT), was determined using Automated Hematology Analyzer Dimension ${ }^{\circledR}$ EXL $^{\text {TM }}$ 200. The serum concentration of FABP4 was quantified using a commercially available enzyme-linked immunosorbent (ELISA) assay kit for FABP4 (CSB-E12995h; Cusabio, Wuhan, China). Briefly, the assay employed the competitive inhibition enzyme immunoassay technique. The microtiter plates provided in the kit had been precoated with FABP4. The assay was conducted according to the manufacturer's instructions. A calibration curve was constructed by plotting the absorbance values at $450 \mathrm{~nm}$ vs. the FABP 4 concentrations of the calibrators, and concentrations of unknown samples were determined by use of this calibration curve. In our study, the lower detection limit (sensitivity) for FABP4 was $15.6 \mathrm{pg} / \mathrm{mL}$, and the detection range was 62.5 $4000 \mathrm{pg} / \mathrm{mL}$. The intra- and interassay coefficient variances in the kit were $<8 \%$ and $<10 \%$, respectively. 
2.3. Assessment of the Cardiovascular Function. One week after blood transfusion, the cardiovascular function was assessed. Echocardiography was performed by a wellexperienced echocardiographer who was blinded to the clinical data, using Vivid S6 equipped with a $1.5-3.6 \mathrm{MHz}$ frequency transducer. Standard two-dimensional and tissue Doppler echocardiography parameters were determined according to the American Society of Echocardiography [31]. Left ventricular end-systolic volume (LVESV) was assessed from apical four-chamber view. Left ventricular ejection fraction (LVEF), fractional shortening (FS), left ventricular mass, and mass index were measured to see left ventricular function. A normal amount of LVEF was considered $>55 \%$, while $>30 \%$ for normal LVFS. Velocities in early $(E)$ and late $(A)$ diastole were recorded. In the age group of 16-40 years old, which included all of our patients, the normal $E / A$ was considered 1.5 (1.0-2.0). Mean values of early $\left(E^{\prime}\right)$ myocardial velocities were calculated to find the $E / E^{\prime}$ ratio. Left atrial volume index (LAVI) and mean pulmonary artery (PA) pressure were also calculated. Tricuspid annular plane systolic excursion (TAPSE) was evaluated to assess right ventricular function (normal values $>20 \mathrm{~cm} / \mathrm{s}$ ). Moreover, 9-lead ECG was recorded and the result was analyzed by the experienced cardiologist.

2.4. Statistical Analysis. Data were analyzed using SPSS 24.0 (SPSS, Chicago, IL, USA). All tests were two-tailed, and $p$ $<0.05$ was considered to be statistically significant, as well as values $<0.1$ as indicating a tendency or trending toward statistical significant. Consideration of $p<0.1$ may be relevant, particularly in a small study [32]. Mean and standard deviation for continuous variables and percentages for categorical variables are the main descriptive statistics reported. Gaussian distribution of the data was assessed by using the Shapiro-Wilk test. Because there was no cut-off value for FABP4, patients were divided into two groups according to median serum FABP4 value to get an equal number of samples for comparison between the lower and higher groups. Discrete variables were compared with the chi-square test (Fisher exact test when appropriate) and continuous variables with Student's $t$-test (Mann-Whitney when appropriate). Pearson correlation coefficients between FABP4 and measurement parameters, including serum ferritin levels and echocardiography parameter (LAVI), were calculated.

\section{Results}

3.1. Patient Characteristics. Table 1 shows the characteristics of the patients with thalassemia major. The mean age was $21.9 \pm 8.0$ years; age range in this study was $15-53$ years; 18 were male; and the mean interval of blood transfusion was $5.7 \pm 7.2$ weeks. The most-reported iron-chelating agent was deferasirox (43.9\%). The mean serum ferritin was $4605.2 \pm 3303.7$, and the mean FABP 4 was $21.6 \pm 1.9 \mathrm{ng} / \mathrm{mL}$.

Clinical characteristics between two groups (higher and lower groups) divided by the median serum FABP4 value $(21.5 \mathrm{ng} / \mathrm{mL})$ are compared as shown in Table 1 . A tendency of difference was observed in serum ferritin levels $(p<0.1)$. There was no gender difference regarding serum FABP4 value in patients with thalassemia major. The presence of siderosis was determined using a cut-off of the serum ferritin as previously described [30]. As shown in Figure 1, patients with siderosis (serum ferritin $>2500 \mathrm{ng} / \mathrm{mL}$ ) have significantly lower levels of FABP4 $(p<0.01)$.

3.2. Liver and Cardiac Function Stratified by FABP4. Table 2 lists the comparison results of liver and cardiac function test stratified by median serum FABP4 value. Concerning the patients with thalassemia major, there were no significant differences in terms of liver function test, including AST levels. Although not reaching statistically significant, ALT tended to decrease in the higher serum FABP4 group compared with the lower one.

Concerning cardiac function, as demonstrated in Table 2, there were significantly lower values of LVEF and LVSV in patients with thalassemia major, with higher serum FABP4 compared with those who have lower serum FABP4 $(63.7 \pm 5.3$ vs. $67.0 \pm 4.8, p<0.05$ and $34.6 \pm 3.9$ vs. $37.1 \pm$ $3.8, p<0.05$, respectively). However, left ventricular posterior wall diastolic diameter (LVPWDD, $9.0 \pm 2.0$ vs. $8.0 \pm$ $1.3, p<0.05)$, LVESV (37.6 \pm 12.7 vs. $29.7 \pm 9.6, p<0.05)$, $E / E^{\prime}(11.1 \pm 3.4$ vs. $8.6 \pm 1.8, p<0.05)$, left ventricular mass index $(109.2 \pm 29.8$ vs. $90.1 \pm 17.0, p<0.05)$, and LAVI $(34.1 \pm 17.1$ vs. $25.8 \pm 5.7, \quad p<0.05)$ were significantly increased in those with higher serum FABP4 levels. In terms of ECG, there were no significant differences in ECG results between the two groups. However, left ventricular hypertrophy (8.7\%), conduction block (8.7), and arrhythmia (4.3\%) were only seen in patients with higher serum FABP4 levels.

3.3. Correlation Coefficients for Serum FABP4 and Liver and Cardiac Function. Figure 2 shows the correlation between FABP4 and serum ferritin. There was a weak negative correlation between serum ferritin and FABP4 $(r=-0.291$, $p<0.05)$. In addition, there was a significantly moderately positive correlation between LAVI and FABP4 $(r=0.316$, $p<0.05)$ in patients with thalassemia major, as shown in Figure 3 .

\section{Discussion}

The effects of iron excess in patients with thalassemia major have remained in increasing attention because of its higher morbidity and mortality in this population. Iron accumulation may increase the production of unstable iron, which can affect reactive oxygen species production and thus perpetuate organ damage [18]. Previous work has demonstrated a favorable inflammatory profile in patients with thalassemia, especially those who developed cardiovascular diseases [33]. Additionally, iron accumulation in tissues may impact its metabolism. The role of FABP4, as one of a novel biomarker for metabolically driven low-grade and chronic inflammation [26], may provide a clue in these mechanisms that occurred in patients with thalassemia major.

Cardiovascular dysfunctions are the most serious problems in patients with thalassemia major. These complications involve fatal cardiac arrhythmias and congestive heart failure $[8,12,14-16]$. The judgment of ventricular 
TABLE 1: Characteristics in total patients and stratified by median of fatty acid-binding protein $4(\mathrm{FABP} 4)$ levels $(\mathrm{median}=21.5 \mathrm{ng} / \mathrm{mL})$.

\begin{tabular}{|c|c|c|c|c|}
\hline & Lower & Higher & Total & $p$ value \\
\hline Age, years $($ mean $\pm S D)$ & $21.8 \pm 7.7$ & $22.0 \pm 8.5$ & $21.9 \pm 8.0$ & 0.929 \\
\hline Sex, $n(\%)$ & & & & 0.592 \\
\hline Male & $9(37.5)$ & $9(37.5)$ & $18(37.5)$ & \\
\hline Female & $15(62.5)$ & $15(62.5)$ & $30(62.5)$ & \\
\hline Age of first transfusion, months (mean \pm SD) & $54.6 \pm 51.9$ & $73.6 \pm 124.1$ & $64.1 \pm 94.6$ & 0.451 \\
\hline Transfusion interval, weeks (mean \pm SD) & $5.2 \pm 2.4$ & $6.3 \pm 10.0$ & $5.7 \pm 7.2$ & 0.187 \\
\hline Type of chelation therapy, $n(\%)$ & & & & 0.968 \\
\hline Deferoxamine monotherapy & $2(9.5)$ & $2(10.0)$ & $4(9.8)$ & \\
\hline Deferiprone monotherapy & $8(38.1)$ & $9(45.0)$ & $17(41.5)$ & \\
\hline Deferasirox monotherapy & $10(47.6)$ & $8(40.0)$ & $18(43.9)$ & \\
\hline Deferoxamine and deferasirox & $1(4.8)$ & $1(5.0)$ & $2(4.9)$ & \\
\hline Pretransfusion $\mathrm{Hb}, \mathrm{g} / \mathrm{dL}($ mean $\pm \mathrm{SD})$ & $7.3 \pm 2.3$ & $7.0 \pm 1.5$ & $7.2 \pm 1.9$ & 0.573 \\
\hline Serum ferritin, $\mathrm{ng} / \mathrm{mL}($ mean $\pm \mathrm{SD})$ & $5436.8 \pm 3689.9$ & $3773.5 \pm 2691.5$ & $4605.2 \pm 3303.7$ & 0.082 \\
\hline $\mathrm{FABP} 4, \mathrm{ng} / \mathrm{mL}($ mean $\pm \mathrm{SD})$ & $20.1 \pm 1.2$ & $23.1 \pm 1.2$ & $21.6 \pm 1.9$ & 0.000 \\
\hline
\end{tabular}

Hb: hemoglobin, Student's $t$-test, Mann-Whitney, or chi-square tests were used as appropriate.

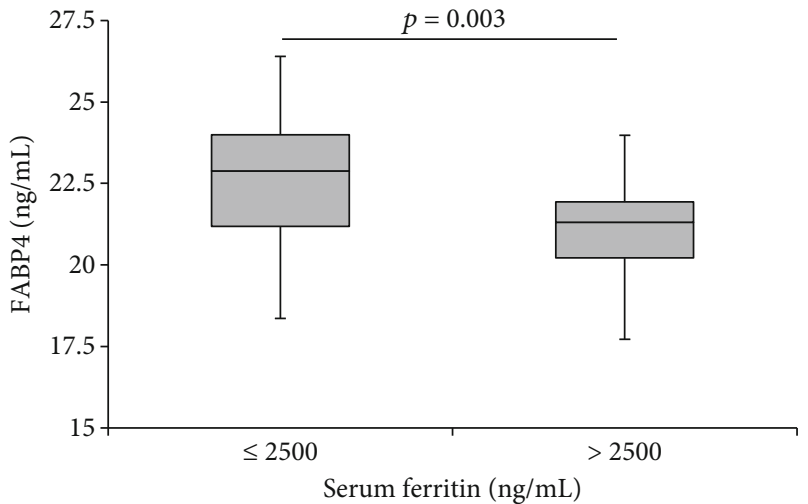

FIGURE 1: Serum fatty acid-binding protein 4 (FABP4) levels in patients with thalassemia major with and without siderosis (serum ferritin $>2500 \mathrm{ng} / \mathrm{mL}$ and $\leq 2500 \mathrm{ng} / \mathrm{mL}$, respectively, $p=0.003)$.

dysfunction in patients with thalassemia major is different from nonanemic patients. Patients with thalassemia major undergo cardiovascular adjustment to chronic anemia, such as high ejection fraction, tachycardia at rest, low blood pressure, and increased end-diastolic volume [13]. Prolonged anemia also induces dyspnea that mimics signs and symptoms of cardiac deterioration. Although serum ferritin is not a sufficient surrogate for cardiac iron evaluation, noninvasive assessment may provide useful information. Mostly, diastolic dysfunction occurred earlier than systolic dysfunction [34].

There is emerging evidence that accumulation of iron may induce lipid peroxidation via oxidative stress, which results in changes of membrane fatty acid. Furthermore, these can impair metabolism and end up with an inflammatory state [35]. Additionally, a growing body of evidence indicates that FABP4 contributes to the inflammatory and metabolic pathway of cardiovascular dysfunction [22, 25, 26].
Studies in nonthalassemia patients showed that women exhibited higher FABP4 levels than men, possibly due to disparities in adipose storage or the contribution of sex hormones in the secretion regulation [36, 37]. Based on our study, the gender differences in serum FABP4 levels were absent in patients with thalassemia major. Supporting this, Vlychou and colleagues found that there was no significant difference in terms of body mass index (BMI) in male and female patients with thalassemia major [38]. Furthermore, the overall fat was lower in patients with thalassemia major compared to nonthalassemia controls [39]. Impairment both in height and in weight concurrently with lower plasma value of essential amino acids occurs in patients with thalassemia [40]. Patients with thalassemia had lower BMI in comparison to healthy controls, which was affected by chronic anemia, ferritin elevation, and noncompliance of iron chelation therapy [39]. Additionally, the conditions of chronic anemia and ineffective erythropoiesis in patients with thalassemia are related to a hypermetabolic state that result in decreased fat and deficiency in fat-soluble vitamins [13].

Left atrial enlargement appears in systolic and diastolic dysfunctions [41]. LAVI, the value of left atrial volume divided by body surface area, is recommended by the American Society of Echocardiography to measure left atrial size [42]. In this study, increased FABP4 levels are correlated with increased LAVI. It may imply that diastolic dysfunction may occur with the increased FABP4 levels. In addition, regarding the left ventricular diastolic function, the $E / E^{\prime}$ ratio was related to increased FABP4 level. Higher FABP4 value has been associated with metabolic syndrome, inflammation, development of atherosclerosis, and insulin resistance $[26,43]$, and thus, various metabolic changes due to iron toxicity $[5,17,18]$ may be accompanied by an increase in FABP4 level and simultaneously promote an increase in LAVI and $E / E^{\prime}$ ratio. However, the cross-sectional design 
TABLE 2: Liver enzyme and cardiac function parameters stratified by median of FABP4 levels (median $=21.5 \mathrm{ng} / \mathrm{mL}$ ).

\begin{tabular}{|c|c|c|c|}
\hline & Lower & Higher & $p$ value \\
\hline \multicolumn{4}{|l|}{ Liver enzymes, $n g / m L($ mean $\pm S D)$} \\
\hline AST & $64.8 \pm 4.9$ & $50.4 \pm 6.3$ & 0.114 \\
\hline ALT & $58.8 \pm 41.2$ & $40.0 \pm 22.0$ & 0.057 \\
\hline \multicolumn{4}{|l|}{ Cardiac function parameters (mean $\pm S D)$} \\
\hline \multicolumn{4}{|l|}{ Echocardiography } \\
\hline LVPWDD (mm) & $8.0 \pm 1.3$ & $9.0 \pm 2.0$ & 0.049 \\
\hline LVESV (mL) & $29.7 \pm 9.6$ & $37.6 \pm 12.7$ & 0.019 \\
\hline LVEF (\%) & $67.0 \pm 4.8$ & $63.7 \pm 5.3$ & 0.029 \\
\hline LVFS (\%) & $37.1 \pm 3.8$ & $34.6 \pm 3.9$ & 0.029 \\
\hline LV mass index & $90.1 \pm 17.0$ & $109.2 \pm 29.8$ & 0.011 \\
\hline $\mathrm{LV}$ diastolic function $E / A$ ratio $(\mathrm{m} / \mathrm{s})$ & $1.6 \pm 0.3$ & $1.6 \pm 0.4$ & 0.849 \\
\hline $\mathrm{LV}$ diastolic function $E / E^{\prime}$ & $8.6 \pm 1.8$ & $11.1 \pm 3.4$ & 0.003 \\
\hline $\mathrm{LV}$ diastolic function LAVI $\left(\mathrm{mL} / \mathrm{m}^{2}\right)$ & $25.8 \pm 5.7$ & $34.1 \pm 17.1$ & 0.040 \\
\hline Mean PA pressure $(\mathrm{mmHg})$ & $17.3 \pm 5.1$ & $22.0 \pm 11.0$ & 0.081 \\
\hline TAPSE (mm) & $22.2 \pm 2.9$ & $23.6 \pm 4.2$ & 0.206 \\
\hline Electrocardiography, $n(\%)$ & & & 0.365 \\
\hline Normal & $12(52.2)$ & $9(39.1)$ & \\
\hline Tachycardia & $3(13.0)$ & $2(8.7)$ & \\
\hline ST-T wave changes & $8(34.8)$ & $7(30.4)$ & \\
\hline Left ventricular hypertrophy & $0(0.0)$ & $2(8.7)$ & \\
\hline Conduction block & $0(0.0)$ & $2(8.7)$ & \\
\hline Arrhythmia & $0(0.0)$ & $1(4.3)$ & \\
\hline
\end{tabular}

AST: aspartate transaminase; ALT: alanine transaminase; LVPWDD: left ventricular posterior wall diastolic diameter; LVESV: left ventricular end-systolic volume; LVEF: left ventricular ejection fraction; LVFS: left ventricular fractional shortening; LV: left ventricle; LAVI: left atrial volume index; PA: pulmonary artery; TAPSE: tricuspid annular plane systolic excursion; Student's $t$-test, Mann-Whitney, or chi-square tests were used as appropriate.

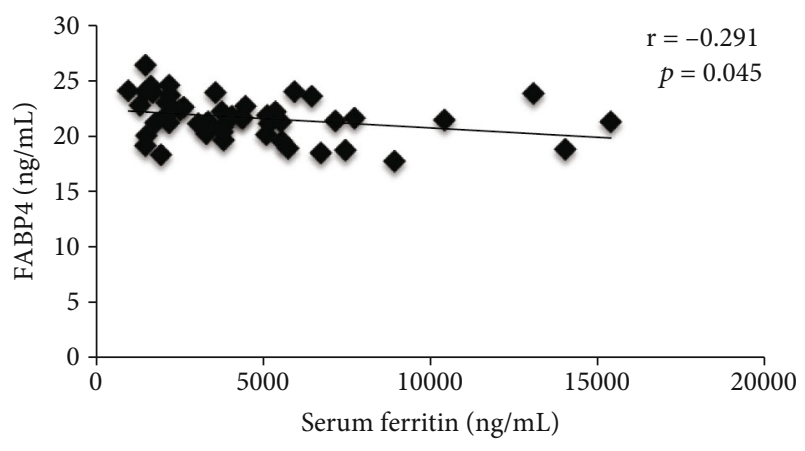

FIGURE 2: Correlation between serum ferritin and FABP4.

of this study prevents the demonstration of the mechanisms by which increased LAVI and $E / E^{\prime}$ ratio are related to FABP4 levels.

This study found that increased left ventricular mass index is associated with increased serum FABP4 levels. A raised FABP4 value has been associated with diabetes mellitus, development of atherosclerosis, inflammation, and insulin resistance; and the iron accumulation in patients with thalassemia major may induce these conditions $[22,26]$. Therefore, those mechanisms may simultaneously promote an increase in the left ventricular mass index. Moreover, in this study, LVEF and LVFS were lower among patients with higher FABP4 levels. In terms of range value of echocardiography parameters, it is important to use the different normal ranges for patients with thalassemia major, because in these patients without cardiac iron overload, LVEDV is increased and LVESV is decreased, which leads to increased LVSV, LVEF, and cardiac output compared to healthy nonanemic controls [4, 13]. Higher LV mass and the right ventricular indexes can be caused by a hyperdynamic state. Therefore, it is challenging to identify ventricular systolic dysfunction in patients with thalassemia major. Hyperdynamic effects related to chronic anemia facilitated to preserve myocardial fiber shortening and normal ejection fraction [44].

Interestingly, abnormalities in ECG especially left ventricular hypertrophy, conduction block, and arrhythmia were only demonstrated in patients with higher FABP4 levels. A previous study showed similar findings of ECG abnormalities in patients with thalassemia [45]. It was suggested that FABP4 may have an essential function in the regulation of cardiac depolarization and arrhythmias [27]. Additionally, patients with thalassemia major have higher chronic inflammation due to iron toxicity [19-21], which can increase the risk of cardiac arrhythmias through mechanisms involving vascular injury, myocardial fibrosis, increased sympathetic activity, and failure of ion channel [27]. 


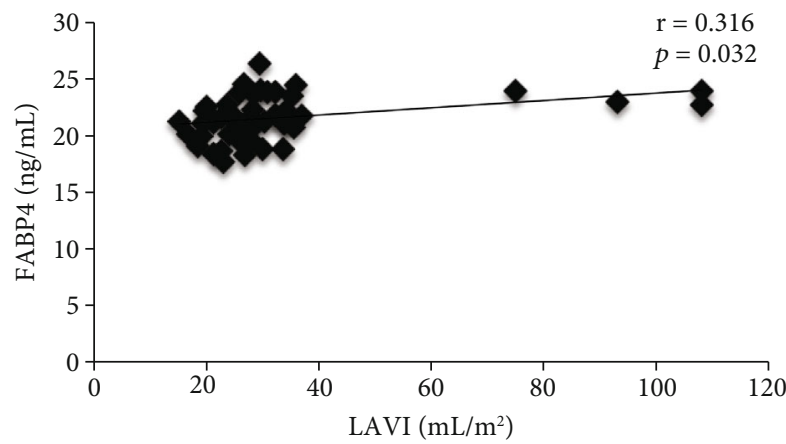

FIgURe 3: Correlation between left atrial volume index (LAVI) and FABP4.

Inverse correlation of serum FABP4 and serum ferritin levels in patients with thalassemia major was demonstrated in our study. This could be related to the toxicity of iron excess in adipocytes that could prevent its role to produce or secrete FABP4. Additionally, it has been described that the levels of hemoglobin and ineffective erythropoiesis were related to hypermetabolic states that affect the amounts of body fat in patients with thalassemia $[13,46]$. Total cholesterol and low-density lipoprotein cholesterol were lower in patients with thalassemia than in control participants [47-49]. The levels of various nutritional biomarkers were also reduced in patients with transfusion-dependent thalassemia [50]. Although previous reports have shown the link between adiposity and FABP4, in other studies, the connection of regulation and disease correlation appears to be complex, especially in rapid loss of adiposity, which might lead to an enormous breakdown of lipid [51]. Therefore, enhanced serum FABP4 levels may still occur in condition of lower adipocyte secretion [48-51].

Higher levels of AST do not always indicate health problem because they could result from some factors such as strenuous physical exercise [9]. However, the ALT level is more specific for hepatocellular damage secondary to iron excess as the liver is the first target organ dysfunction. In this study, ALT tended to increase in patients with lower serum FABP4. This could be under the aforementioned mechanism of concomitant iron toxicity in the liver and adipocytes.

In line with previous work, this study has demonstrated a favorable inflammatory profile in patients with thalassemia, especially those who developed cardiovascular dysfunction [33]. In this study, although not classified into abnormalities, patients with thalassemia who presented reduced cardiac function based on echocardiography and ECG showed higher levels of serum FABP4. The role of FABP4, as one of a novel biomarker for metabolically driven low-grade and chronic inflammation [26], may provide a clue in these mechanisms that occurred in patients with thalassemia major. In addition, a study by $\mathrm{Tu}$ and colleagues showed that FABP4 is a prognostic biomarker in stroke patients [52]. Based on this study, for further investigation, it is also possible for FABP4 to be a prognostic biomarker for cardiovascular complications in patients with thalassemia because the deterioration of cardiac function in patients with thalassemia may occur progressively due to cardiac iron accumulation. This emphasized the importance of optimal iron-chelation therapy. Therefore, problems related to nonoptimal iron-chelation therapy such as noncompliance, financial problem including health insurance should be managed well.

Although this is the first study that sought to explore the correlation between FABP4 and cardiac function in patients with thalassemia major, several limitations need to be considered in the interpretation of the study findings. Data on hemoglobin typing and genetic analysis were not obtained. The height and weight (and consequently the BMI) of the patients were not obtained in this study. In addition, the parameters related to metabolism were not examined. In line with this, the measurement of inflammatory markers could potentially reveal an association between metabolic and inflammatory status in patients with thalassemia. However, estimation of iron overload was conducted using serum ferritin because of its affordability and availability; despite that, T2* magnetic resonance imaging (MRI) is the recommended method to calculate iron accumulation [53]. Single cross-sectional ferritin measurement may be misrepresentative because it may not reflect long-term ferritin levels [13]. A prospective study with a greater number of subjects and even comparison to healthy subjects is necessary for any further investigation of FABP4 in patients with thalassemia. Additionally, the inclusion of more patients with cardiac abnormalities and comparison with the gold standard of iron toxicity examination (T2* MRI) are important to make further diagnostic analysis and also adjustment of confounding factor in order to see possibility of FABP4 as a potential biomarker of cardiac iron toxicity.

\section{Conclusions}

There were correlations between serum FABP4 and cardiac function, as well as serum ferritin in patients with thalassemia major. FABP4 levels could be a potentially useful clinical biomarker for cardiac dysfunction via metabolic and inflammatory pathways due to iron accumulation and toxicity in patients with thalassemia major. Further prospective studies are needed to elucidate whether increased serum FABP4 level is a marker or a potential mechanism for iron-related cardiac involvement in patients with thalassemia major.

\section{Data Availability}

The data used to support the findings of this study are available from the corresponding author upon request.

\section{Conflicts of Interest}

The authors declare that there are no conflicts of interest.

\section{Acknowledgments}

The authors greatly appreciate the cooperation of the members of the Thalassemia Parents Association who participated in this investigation. This research was supported by the Academic Leadership Grant (ALG) for Thalassemia Study and Internal Research Grant of Universitas Padjadjaran. In 
addition, this research was also partially funded by the Indonesian Ministry of Research, Technology, and Higher Education under World Class University (WCU) Program managed by Institut Teknologi Bandung.

\section{References}

[1] M. Angastiniotis and S. Lobitz, "Thalassemias: an overview," International Journal of Neonatal Screening, vol. 5, no. 1, p. 16, 2019.

[2] V. De Sanctis, C. Kattamis, D. Canatan et al., “ $\beta$-Thalassemia distribution in the old world: an ancient disease seen from a historical standpoint," Mediterranean Journal of Hematology and Infectious Diseases, vol. 9, no. 1, article e2017018, 2016.

[3] D. Rund and E. Rachmilewitz, " $\beta$-Thalassemia," The New England Journal of Medicine, vol. 353, no. 11, pp. 1135-1146, 2005.

[4] T. Cogliandro, G. Derchi, L. Mancuso et al., "Guideline recommendations for heart complications in thalassemia major," Journal of Cardiovascular Medicine, vol. 9, no. 5, pp. 515525, 2008.

[5] Y. Kohgo, K. Ikuta, T. Ohtake, Y. Torimoto, and J. Kato, "Body iron metabolism and pathophysiology of iron overload," International Journal of Hematology, vol. 88, no. 1, pp. 7-15, 2008.

[6] J. B. Porter, "Pathophysiology of transfusional iron overload: contrasting patterns in thalassemia major and sickle cell disease," Hemoglobin, vol. 33, supplement 1, pp. S37-S45, 2009.

[7] E. Poggiali, E. Cassinerio, L. Zanaboni, and M. D. Cappelini, "An update on iron chelation therapy," Blood Transfusion, vol. 10, no. 4, pp. 411-422, 2012.

[8] S. Fucharoen and P. Winichagoon, "Haemoglobinopathies in Southeast Asia," The Indian Journal of Medical Research, vol. 134, no. 4, pp. 498-506, 2011.

[9] M. Y. Abdalla, M. Fawzi, S. R. Al-Maloul, N. El-Banna, R. F. Tayyem, and I. M. Ahmad, "Increased oxidative stress and iron overload in Jordanian $\beta$-thalassemic children," Hemoglobin, vol. 35, no. 1, pp. 67-79, 2011.

[10] C. Sengsuk, O. Tangvarasittichai, P. Chantanaskulwong et al., "Association of iron overload with oxidative stress, hepatic damage and dyslipidemia in transfusion-dependent $\beta$-thalassemia/HbE patients," Indian Journal of Clinical Biochemistry, vol. 29, no. 3, pp. 298-305, 2014.

[11] H. Farhangi, Z. Badiei, H. M. Moghaddam, and M. R. Keramati, "Assessment of heart and liver iron overload in thalassemia major patients using T2* magnetic resonance imaging," Indian Journal of Hematology and Blood Transfusion, vol. 33, no. 2, pp. 228-234, 2017.

[12] S. Lekawanvijit and N. Chattipakorn, "La myocardiopathie thalassemique par surcharge en fer : l'evaluation de la quantite de fer et des mecanismes de perturbation mecanique et electrique causes par la toxicite ferreuse," The Canadian Journal of Cardiology, vol. 25, no. 4, pp. 213-218, 2009.

[13] D. J. Pennell, J. E. Udelson, A. E. Arai et al., "Cardiovascular function and treatment in $\beta$-thalassemia major," Circulation, vol. 128, no. 3, pp. 281-308, 2013.

[14] Y. Aydinok, J. B. Porter, A. Piga et al., "Prevalence and distribution of iron overload in patients with transfusion- dependent anemias differs across geographic regions: results from the CORDELIA study," European Journal of Haematology, vol. 95, no. 3, pp. 244-253, 2015.
[15] R. D. Javier, F. Zapata-Mesina, and M. R. I. D. Castillo, “Tissue Doppler echocardiography in detection of myocardial iron overload confirmed by cardiac MRI in patients with beta thalassemia major: a meta-analysis," Journal of Hematology, vol. 3, no. 1, pp. 1-9, 2014.

[16] A. K. Kondur, T. Li, P. Vaitkevicius, and L. Afonso, "Quantification of myocardial iron overload by cardiovascular magnetic resonance imaging T2* and review of the literature," Clinical Cardiology, vol. 32, no. 6, pp. E55-E59, 2009.

[17] R. S. Britton, K. L. Leicester, and B. R. Bacon, "Iron toxicity and chelation therapy," International Journal of Hematology, vol. 76, no. 3, pp. 219-228, 2002.

[18] C. Lehmann, S. Islam, S. Jarosch et al., "The utility of iron chelators in the management of inflammatory disorders," Mediators of Inflammation, vol. 2015, Article ID 516740, 2015.

[19] Y. Enli, Y. I. Balci, C. Gönen, E. Uzun, and A. Polat, "Adipocytokine concentrations in children with different types of betathalassemia," Scandinavian Journal of Clinical and Laboratory Investigation, vol. 74, no. 4, pp. 306-311, 2014.

[20] K. I. Elsayh, W. S. Mohammed, A. M. Zahran, and K. Saad, "Leukocytes apoptosis and adipocytokines in children with beta thalassemia major," Clinical and Experimental Medicine, vol. 16, no. 3, pp. 345-350, 2016.

[21] N. Chaliasos, A. Challa, E. Hatzimichael et al., "Serum adipocytokine and vascular inflammation marker levels in betathalassaemia major patients," Acta Haematologica, vol. 124, no. 4, pp. 191-196, 2010.

[22] T. Fuseya, M. Furuhashi, S. Yuda et al., "Elevation of circulating fatty acid-binding protein 4 is independently associated with left ventricular diastolic dysfunction in a general population," Cardiovascular Diabetology, vol. 13, no. 1, p. 126, 2014.

[23] M. Furuhashi and G. S. Hotamisligil, "Fatty acid-binding proteins: role in metabolic diseases and potential as drug targets," Nature Reviews. Drug Discovery, vol. 7, no. 6, pp. 489-503, 2008.

[24] M. Furuhashi, S. Ishimura, H. Ota, and T. Miura, "Lipid chaperones and metabolic inflammation," International Journal of Inflammation, vol. 2011, Article ID 642612, 2011.

[25] M. Furuhashi, S. Saitoh, K. Shimamoto, and T. Miura, "Fatty acid-binding protein 4 (FABP4): pathophysiological insights and potent clinical biomarker of metabolic and cardiovascular diseases," Clinical Medicine Insights. Cardiology, vol. 8, supplement 3, pp. 23-33, 2014.

[26] M. Furuhashi, "Fatty acid-binding protein 4 in cardiovascular and metabolic diseases," Journal of Atherosclerosis and Thrombosis, vol. 26, no. 3, pp. 216-232, 2019.

[27] C.-P. Wang, C.-C. Hsu, W.-C. Hung et al., "Plasma fatty acidbinding protein 4 (FABP4) level is associated with abnormal QTc interval in patients with stable angina and chronic kidney disease," BMC Cardiovascular Disorders, vol. 19, no. 1, p. 153, 2019.

[28] I. Shahramian, E. Akhlaghi, A. Ramezani, A. Rezaee, N. Noori, and E. Sharafi, "A study of leptin serum concentrations in patients with major beta-thalassemia," Iranian Journal of Pediatric Hematology Oncology, vol. 3, no. 2, pp. 59-63, 2013.

[29] S. Crippa, V. Rossella, A. Aprile et al., "Bone marrow stromal cells from $\beta$-thalassemia patients have impaired hematopoietic supportive capacity," The Journal of Clinical Investigation, vol. 129, no. 4, pp. 1566-1580, 2019.

[30] A. Taher and A. N. Saliba, "Iron overload in thalassemia: different organs at different rates," Hematology, vol. 2017, no. 1, pp. 265-271, 2017. 
[31] S. F. Nagueh, O. A. Smiseth, C. P. Appleton et al., "Recommendations for the evaluation of left ventricular diastolic function by echocardiography: an update from the American Society of Echocardiography and the European Association of Cardiovascular Imaging," Journal of the American Society of Echocardiography, vol. 29, no. 4, pp. 277-314, 2016.

[32] M. S. Thiese, B. Ronna, and U. Ott, "P value interpretations and considerations," Journal of Thoracic Disease, vol. 8, no. 9, pp. E928-E931, 2016.

[33] A. I. Triantafyllou, D. T. Farmakis, K. M. Lampropoulos et al., "Impact of $\beta$-thalassemia trait carrier state on inflammatory status in patients with newly diagnosed hypertension," Journal of Cardiovascular Medicine, vol. 20, no. 5, pp. 284-289, 2019.

[34] L. Mancuso, A. Vitrano, A. Mancuso, M. Sacco, A. Ledda, and A. Maggio, "Left ventricular diastolic dysfunction in $\beta$-thalassemia major with heart failure," Hemoglobin, vol. 42, no. 1, pp. 68-71, 2018.

[35] U. Ahmed, P. S. Latham, and P. S. Oates, "Interactions between hepatic iron and lipid metabolism with possible relevance to steatohepatitis," World Journal of Gastroenterology, vol. 18, no. 34, pp. 4651-4658, 2012.

[36] K. J. Prentice, J. Saksi, and G. S. Hotamisligil, "Adipokine FABP4 integrates energy stores and counterregulatory metabolic responses," Journal of Lipid Research, vol. 60, no. 4, pp. 734-740, 2019.

[37] X. Zhang, D. Li, H. Wang, C. Pang, Y. Wu, and F. Wen, "Gender difference in plasma fatty-acid-binding protein 4 levels in patients with chronic obstructive pulmonary disease," Bioscience Reports, vol. 36, no. 1, article e00302, 2016.

[38] M. Vlychou, E. Alexiou, P. Thriskos, I. Fezoulidis, and K. Vassiou, "Body composition in adult patients with thalassemia major," International Journal of Endocrinology, vol. 2016, Article ID 6218437, 2016.

[39] H. Hammod, T. A. Mokif, and H. J. Al-Harbi, "The correlation between thalassemia with body mass index and blood groups in children and adult patient in the province of Babylon, Iraq," Asian Journal of Pharmaceutical and Clinical Research, vol. 11, no. 9, pp. 509-512, 2018.

[40] Y. M. Abdulrazzaq, A. Ibrahim, A. I. Al-Khayat, and K. Dawson, " $\beta$-Thalassemia major and its effect on amino acid metabolism and growth in patients in the United Arab Emirates," Clinica Chimica Acta, vol. 352, no. 1-2, pp. 183-190, 2005.

[41] B. Ristow, S. Ali, M. A. Whooley, and N. B. Schiller, "Usefulness of left atrial volume index to predict heart failure hospitalization and mortality in ambulatory patients with coronary heart disease and comparison to left ventricular ejection fraction (from the Heart and Soul Study)," The American Journal of Cardiology, vol. 102, no. 1, pp. 70-76, 2008.

[42] R. M. Lang, M. Bierig, R. B. Devereux et al., "Recommendations for chamber quantification: a report from the American Society of Echocardiography's guidelines and standards committee and the chamber quantification writing group, developed in conjunction with the European Association of Echocardiography, a branch of the European Society of Cardiology," Journal of the American Society of Echocardiography, vol. 18, no. 12, pp. 1440-1463, 2005.

[43] A. Cabre, P. Valdovinos, I. Lazaro, G. Bonet, A. Bardaji, and L. Masana, "Parallel evolution of circulating FABP4 and NTproBNP in heart failure patients," Cardiovascular Diabetology, vol. 12, no. 1, 2013.
[44] A. A. Abbas, B. Najeb, A. Abdulhussein et al., "Echocardiographic parameters of left ventricle systolic and diastolic function in patients with $\beta$-thalassemia major," The Iraqi Postgraduate Medical Journal, vol. 11, no. 4, pp. 562-568, 2012.

[45] F. H. Al-Lami and M. Q. Hussein, "Electrocardiographic changes among beta-thalassemic major patients in Ibn AlBaladi thalassemia center-Baghdad," Al-Kindy College Medical Journal, vol. 9, no. 1, pp. 36-42, 2013.

[46] G. Dedoussis, M. C. Kyrtsonis, N. Andrikopoulos, E. Voskaridou, and A. Loutradis, "Inverse correlation of plasma leptin and soluble transferrin receptor levels in betathalassemia patients," Annals of Hematology, vol. 81, no. 9, pp. 543-547, 2002.

[47] S. Haghpanah, M. Davani, B. Samadi, A. Ashrafi, and M. Karimi, "Serum lipid profiles in patients with betathalassemia major and intermedia in southern Iran," Journal of Research in Medical Sciences, vol. 15, no. 3, pp. 150-154, 2010.

[48] K. M. Mansi and T. A. Aburjai, "Lipid profile in Jordanian children with ß-thalassemia major," International Journal of Hematology and Oncology, vol. 18, no. 2, pp. 93-98, 2008.

[49] S. M. Ragab, M. A. Safan, and A. S. Sherif, "Lipid profiles in $\beta$ thalassemic children," Menoufia Medical Journal, vol. 27, no. 1, pp. 66-72, 2014.

[50] L. M. Sherief, A. El-Salam, M. Sanaa et al., "Nutritional biomarkers in children and adolescents with beta-thalassemiamajor: an Egyptian center experience," BioMed Research International, vol. 2014, Article ID 261761, 2014.

[51] G. S. Hotamisligil and D. A. Bernlohr, "Metabolic functions of FABPs-mechanisms and therapeutic implications," Nature Reviews. Endocrinology, vol. 11, no. 10, pp. 592-605, 2015.

[52] W.-J. Tu, X.-W. Zeng, A. Deng et al., "Circulating FABP4 (fatty acid-binding protein 4) is a novel prognostic biomarker in patients with acute ischemic stroke," Stroke, vol. 48, no. 6 , pp. 1531-1538, 2017.

[53] J. C. Wood, "Estimating tissue iron burden: current status and future prospects," British Journal of Haematology, vol. 170, no. 1, pp. 15-28, 2015. 